\title{
Passivity Preserving Model Reduction via Interpolation of Spectral Zeros*
}

\author{
D.C. SORENSEN ${ }^{\dagger}$ \\ e-mail: sorensen@rice.edu
}

December 20, 2002

\begin{abstract}
An algorithm is developed for passivity preserving model reduction of linear time invariant systems. Implementation schemes are described for both medium scale (dense) and large scale (sparse) applications. The algorithm is based upon interpolation at selected spectral zeros of the original transfer function to produce a reduced transfer function that has the specified roots as its spectral zeros. These interpolation conditions are satisfied through the computation of a basis for a selected invariant subspace of a certain blocked matrix which has the spectral zeros as its spectrum. Explicit interpolation is avoided and passivity of the reduced model is established, instead, through satisfaction of the necessary conditions of the Positive Real Lemma. It is also shown that this procedure indirectly solves the associated controllability and observability Riccati equations and how to select the interpolation points to give maximal or minimal solutions of these equations. From these, a balancing transformation may be constructed to give a reduced model that is balanced as well as passive and stable.
\end{abstract}

\section{Introduction}

This paper is concerned with linear time invariant (LTI) systems

$$
\boldsymbol{\Sigma}: \quad \dot{\mathbf{x}}(t)=\mathbf{A x}(t)+\mathbf{B u}(t), \quad \mathbf{y}(t)=\mathbf{C x}(t)+\mathbf{D} \mathbf{u}(t),
$$

where $\mathbf{A} \in \mathbb{R}^{n \times n}, \mathbf{B} \in \mathbb{R}^{n \times m}, \mathbf{C} \in \mathbb{R}^{p \times n}, \mathbf{D} \in \mathbb{R}^{p \times m}$. These systems arise frequently in many branches of engineering. Circuit simulation is an important example and in this application the system $\boldsymbol{\Sigma}$ is often passive. Model reduction with a passivity constraint is of great importance in this context and has been studied by many researchers, including Rohrer [18], Ober [17], Feldmann, Freund, and Bai [10], [11], [4], [5], [6] Gugercin and Antoulas [13] and others.

The concept of passivity is of general interest in control theory. A system is passive if it does not generate energy internally, and strictly passive if it consumes or dissipates input energy. A primary example of a passive system is an RLC circuit consisting only of resistors, conductors, and capacitors. Typically, in circuit simulation, one is interested in replacing a component described by a large linear circuit with one of much lower order for purposes of speeding up the simulation. Obviously, it is important to produce a reduced model that preserves important system properties and response characteristics such as stability and passivity.

Projection methods construct matrices $\mathbf{V} \in \mathbb{R}^{n \times k}$ and $\mathbf{W} \in \mathbb{R}^{n \times k}$ such that $\mathbf{W}^{T} \mathbf{V}=\mathbf{I}_{k}$ that are used to obtain a reduced model

$$
\hat{\mathbf{\Sigma}}: \quad \dot{\hat{\mathbf{x}}}(t)=\hat{\mathbf{A}} \hat{\mathbf{x}}(t)+\hat{\mathbf{B}} \mathbf{u}(t), \quad \hat{\mathbf{y}}(t)=\hat{\mathbf{C}} \hat{\mathbf{x}}(t)+\mathbf{D u}(t),
$$

\footnotetext{
${ }^{*}$ This work was supported in part by the NSF through Grants CCR-9988393 and ACI-0082645.

${ }^{\dagger}$ Department of Computational and Applied Mathematics, MS 134, Rice University, Houston, Texas 77251-1892.
} 
where

$$
\hat{\mathbf{A}}=\mathbf{W}^{T} \mathbf{A V}, \quad \hat{\mathbf{B}}=\mathbf{W}^{T} \mathbf{B}, \quad \hat{\mathbf{C}}=\mathbf{C V} .
$$

A new projection method is developed here that preserves both stability and passivity. This method is quite novel because it obtains the projection matrices $\mathbf{V}, \mathbf{W}$ as a by-product of a certain eigenvalue problem. These matrices are constructed from a basis set for an invariant subspace associated with that problem. The derivation is motivated by the important recent work of Antoulas [2] characterizing passivity through interpolation conditions.

This approach grew out of a desire to satisfy these interpolation conditions indirectly with a computational scheme that would be suitable for large scale computation. This scheme, based upon calculation of an invariant subspace, can be viewed as a means to constructively satisfy the sufficiency conditions of the Positive Real Lemma (generally known as the Yakubovich-Kalman-Popov-Anderson Lemma, see Chap. 5 of [3] or Chap. 13 of [20] and original references therein). This derivation is given in Section 2. In Section 3, it is shown that the scheme also indirectly constructs solutions to the standard Riccati equations associated with this problem and easily satisfied conditions are given to obtain minimal (or maximal) solutions of these equations. From these, a positive real balancing transformation can be constructed. Finally, in Section 4, algorithms for both dense and large (sparse) problems are given. The results of a computational example involving an RLC "ladder circuit" are discussed in both settings. These results indicate that the new approach is very promising.

Generally, in this paper, upper case bold letters $(\mathbf{A}, \mathbf{B}$, etc. $)$ will denote matrices, lower case bold letters $(\mathbf{x}, \mathbf{y}$, etc. $)$ will denote vectors, and non-bold or Greek letters will denote scalars. Occasionally, script letters $(\mathcal{A}, \mathcal{E}$, etc. $)$ will be used to denote special or structured matrices. Conjugate transpose is denoted by $\mathbf{A}^{*}$ and transpose by $\mathbf{A}^{T}$.

\section{Model Reduction of Passive Systems}

Throughout the remainder of this discussion, it shall be assumed that $m=p$, i.e., that $\mathbf{B} \in \mathbb{R}^{n \times p}, \mathbf{C} \in \mathbb{R}^{p \times n}$. Moreover, the matrix $\mathbf{A}$ is assumed to be stable ( the spectrum $\sigma(\mathbf{A})$ is contained in the open left half-plane), and that the system $\boldsymbol{\Sigma}$ is both observable and controllable. Finally, it is assumed that the system is passive and that $\mathcal{D}:=\mathbf{D}+\mathbf{D}^{T}$ is positive definite so that $\mathcal{D}=\mathbf{W}_{o}^{T} \mathbf{W}_{o}$ with $\mathbf{W}_{o}$ nonsingular. The transfer function for $\boldsymbol{\Sigma}$ is denoted by $\mathbf{G}(s)=\mathbf{C}\left(s \mathbf{I}_{n}-\mathbf{A}\right)^{-1} \mathbf{B}+\mathbf{D}$.

Passive Systems: Informally, as mentioned previously, a system $\boldsymbol{\Sigma}$ is passive if it cannot produce energy and strictly passive if it consumes energy. Formally, $\boldsymbol{\Sigma}$ is

Passive if $\operatorname{Re} \int_{-\infty}^{t} \mathbf{u}(\tau)^{T} \mathbf{y}(\tau) \mathrm{d} \tau \geq 0$ for all $t \in \mathbb{R}$ and all $\mathbf{u} \in \mathcal{L}_{2}(\mathbb{R})$,

Strictly Passive if there is a $\delta>0$ such that $\operatorname{Re} \int_{-\infty}^{t} \mathbf{u}(\tau)^{T} \mathbf{y}(\tau) \mathrm{d} \tau \geq \delta \int_{-\infty}^{t} \mathbf{u}(\tau)^{T} \mathbf{u}(\tau) \mathrm{d} \tau$ for all $t \in \mathbb{R}$ and all $\mathbf{u} \in \mathcal{L}_{2}(\mathbb{R})$.

There is an equivalent condition for LTI systems that is more easily verified.

Positive Real: The system $\boldsymbol{\Sigma}$ is passive if and only if its transfer function $\mathbf{G}(s)$ is positive real, which means that:

(1) $\mathbf{G}(s)$ is analytic for $\operatorname{Re}(s)>0$ ),

(2) $\operatorname{Re} \mathbf{G}(s) \geq \mathbf{0}$ for $\operatorname{Re}(s)>0$.

Property (2) implies the existence of a stable rational matrix function $\mathbf{W}(s)$ (with stable inverse) such that $\mathbf{G}(s)+$ $\mathbf{G}^{T}(-s)=\mathbf{W}(s) \mathbf{W}^{T}(-s)$. This is the spectral factorization of $\mathbf{G}$ and the quantity $\mathbf{W}$ is a spectral factor of $\mathbf{G}$. The zeros of $\mathbf{W}$, i.e. $\lambda_{i}, i=1, \cdots, n$, such that $\operatorname{det} \mathbf{W}\left(\lambda_{i}\right)=0$, are the spectral zeros of $\mathbf{G}$.

Let

$$
\mathcal{A}:=\left[\begin{array}{ccc}
\mathbf{A} & & \mathbf{B} \\
& -\mathbf{A}^{T} & -\mathbf{C}^{T} \\
\mathbf{C} & \mathbf{B}^{T} & \mathbf{D}+\mathbf{D}^{T}
\end{array}\right] \text { and } \mathcal{E}:=\left[\begin{array}{ccc}
\mathbf{I} & & \\
& \mathbf{I} & \\
& & \mathbf{0}
\end{array}\right]
$$


Then the spectral zeros of $\mathbf{G}$ are the set of all (finite) complex numbers $\lambda$ such that

$$
\operatorname{Rank}(\mathcal{A}-\lambda \mathcal{E})<2 n+p,
$$

i.e. the finite generalized eigenvalues $\sigma(\mathcal{A}, \mathcal{E})$. The set of spectral zeros shall be denoted as $\mathcal{S}_{\mathbf{G}}$. It is easily seen that $\lambda \in \mathcal{S}_{\mathbf{G}} \Rightarrow-\bar{\lambda} \in \mathcal{S}_{\mathbf{G}}$ since $\mathcal{A} \mathbf{q}=\mathcal{E} \mathbf{q} \lambda \Rightarrow \tilde{\mathbf{q}}^{T} \mathcal{A}=(-\bar{\lambda}) \tilde{\mathbf{q}}^{T} \mathcal{E}$, where $\mathbf{q}^{*}:=\left[\mathbf{x}^{*}, \mathbf{y}^{*}, \mathbf{z}^{*}\right]$, and $\tilde{\mathbf{q}}^{*}:=\left[\mathbf{y}^{*},-\mathbf{x}^{*}, \mathbf{z}^{*}\right]$.

Remark on Descriptor Systems: Often, systems are in descriptor form $\mathbf{E} \dot{\mathbf{x}}(t)=\mathbf{A x}(t)+\mathbf{B u}(t), \quad \mathbf{y}(t)=$ $\mathbf{C x}(t)+\mathbf{D u}(t)$, with $\mathbf{E}$ symmetric and positive (semi) definite. When $\mathbf{E}$ is definite, the following derivation is valid essentially without change if $\mathbf{I}$ is replaced by $\mathbf{E}$ in the definition of $\mathcal{E}$. Moreover, this derivation will never require inversion of $\mathbf{E}$ and thus is at least formally valid for the semi-definite case as well.

Now, suppose a reduced model $\hat{\boldsymbol{\Sigma}}$ as defined in (1.1) has been obtained and let $\hat{\mathbf{G}}(s):=\hat{\mathbf{C}}(s \mathbf{I}-\hat{\mathbf{A}})^{-1} \hat{\mathbf{B}}$ be the reduced transfer function. It is desirable to place conditions on this reduced system that provide for the inheritance of passivity from the original system. The approach taken here is entirely motivated by the following theorem of Antoulas proved in [2] that is stated here in a form that is restricted to the problem at hand. This important result indicates that a passive reduced model will result if certain of the spectral zeros are preserved (interpolated) in the reduced model. For real systems, $\mathcal{S}_{\hat{\mathrm{G}}}$ must include conjugate pairs of spectral zeros as well as their reflections across the real axis.

Theorem 2.1 (Antoulas) If $\mathcal{S}_{\hat{\mathbf{G}}} \subset \mathcal{S}_{\mathbf{G}}$ and $\hat{\mathbf{G}}(\lambda)=\mathbf{G}(\lambda)$ for all $\lambda \in \mathcal{S}_{\hat{\mathbf{G}}}$, then the reduced system $\hat{\boldsymbol{\Sigma}}$ is both stable and passive.

Instead of attempting to satisfy these interpolation conditions directly, the approach taken here is to achieve them indirectly through the construction of a basis for a selected invariant subspace of the pair $(\mathcal{A}, \mathcal{E})$. Suppose $\mathcal{A} \mathbf{Q}=\mathcal{E} \mathbf{Q R}$ is a partial real Schur decomposition for the pair $(\mathcal{A}, \mathcal{E})$. Thus, $\mathbf{Q}^{T} \mathbf{Q}=\mathbf{I}$ and $\mathbf{R}$ is real and quasi-upper triangular. Let $\mathbf{Q}^{T}=\left[\mathbf{X}^{T}, \mathbf{Y}^{T}, \mathbf{Z}^{T}\right]$ be partitioned in accordance with the block structure of $\mathcal{A}$. Then

$$
\left[\begin{array}{ccc}
\mathbf{A} & & \mathbf{B} \\
& -\mathbf{A}^{T} & -\mathbf{C}^{T} \\
\mathbf{C} & \mathbf{B}^{T} & \mathbf{D}+\mathbf{D}^{T}
\end{array}\right]\left[\begin{array}{c}
\mathbf{X} \\
\mathbf{Y} \\
\mathbf{Z}
\end{array}\right]=\left[\begin{array}{c}
\mathbf{X} \\
\mathbf{Y} \\
\mathbf{0}
\end{array}\right] \mathbf{R} .
$$

In the following discussion, projections will be constructed from $\mathbf{X}$ and $\mathbf{Y}$ and a reduced model will be obtained from these. The interpolation conditions of Theorem (2.1) do not appear explicitly. In fact, the derivation and the verification that the reduced model is passive and stable is obtained by demonstrating that the sufficiency conditions for the Positive Real Lemma are satisfied by the reduced system. To begin the derivation, it will be useful to have the following two lemmas.

Lemma 2.1 Suppose that $\mathbf{R}$ in (2.2) satisfies $\operatorname{Re}(\lambda)>0$ for all $\lambda \in \sigma(\mathbf{R})$. Then $\mathbf{X}^{T} \mathbf{Y}=\mathbf{Y}^{T} \mathbf{X}$ is symmetric.

Proof: Observe that $\mathcal{A} \mathbf{Q}=\mathcal{E} \mathbf{Q R} \Rightarrow \hat{\mathbf{Q}}^{T} \mathcal{A}=\left(-\mathbf{R}^{T}\right) \hat{\mathbf{Q}}^{T} \mathcal{E}$, where $\hat{\mathbf{Q}}^{T}=\left[\mathbf{Y}^{T},-\mathbf{X}^{T}, \mathbf{Z}^{T}\right]$.

This gives

$$
\hat{\mathbf{Q}}^{T} \mathcal{E} \mathbf{Q R}=\hat{\mathbf{Q}}^{T} \mathcal{A} \mathbf{Q}=-\mathbf{R}^{T} \hat{\mathbf{Q}}^{T} \mathcal{E} \mathbf{Q} .
$$

Since $\hat{\mathbf{Q}}^{T} \mathcal{E} \mathbf{Q}=\mathbf{Y}^{T} \mathbf{X}-\mathbf{X}^{T} \mathbf{Y}$, it follows that

$$
\mathbf{R}^{T}\left(\mathbf{Y}^{T} \mathbf{X}-\mathbf{X}^{T} \mathbf{Y}\right)+\left(\mathbf{Y}^{T} \mathbf{X}-\mathbf{X}^{T} \mathbf{Y}\right) \mathbf{R}=\mathbf{0}
$$

Hence, $\mathbf{Y}^{T} \mathbf{X}-\mathbf{X}^{T} \mathbf{Y}=\mathbf{0}$ is the unique solution to this Lyapunov equation under the assumption on $\sigma(\mathbf{R})$ and the lemma is proved.

The matrices $\mathbf{X}$ and $\mathbf{Y}$ will be used to construct the matrices $\mathbf{W}$ and $\mathbf{V}$ with $\mathbf{W}^{T} \mathbf{V}=\mathbf{I}$. In this construction, it will be useful to know something about the ranks of $\mathbf{X}$ and $\mathbf{Y}$. 
Lemma 2.2 If $\mathbf{X}, \mathbf{Y}, \mathbf{Z}, \mathbf{R}$ are as specified in equation (2.2), then $\mathbf{X}$ and $\mathbf{Y}$ are both full rank. Moreover,

$$
\begin{aligned}
\mathbf{X}^{T} \mathbf{A}^{T} \mathbf{Y}+\mathbf{Y}^{T} \mathbf{A} \mathbf{X} & =\mathbf{Z}^{T} \mathcal{D} \mathbf{Z} \\
\mathbf{C X}+\mathbf{B}^{T} \mathbf{Y} & =-\mathcal{D} \mathbf{Z} .
\end{aligned}
$$

Proof: From (2.2), it follows that

$$
\begin{aligned}
\mathbf{A X}+\mathbf{B Z} & =\mathbf{X R} \\
\mathbf{A}^{T} \mathbf{Y}+\mathbf{C}^{T} \mathbf{Z} & =-\mathbf{Y R} \\
\mathbf{C X}+\mathbf{B}^{T} \mathbf{Y}+\mathcal{D} \mathbf{Z} & =\mathbf{0}
\end{aligned}
$$

Hence,

$$
\begin{aligned}
\mathbf{Y}^{T} \mathbf{A X}+\mathbf{Y}^{T} \mathbf{B Z} & =\left(\mathbf{Y}^{T} \mathbf{X}\right) \mathbf{R} \\
\mathbf{X}^{T} \mathbf{A}^{T} \mathbf{Y}+\mathbf{X}^{T} \mathbf{C}^{T} \mathbf{Z} & =-\left(\mathbf{X}^{T} \mathbf{Y}\right) \mathbf{R} \\
\mathbf{Z}^{T} \mathbf{C X}+\mathbf{Z}^{T} \mathbf{B}^{T} \mathbf{Y} & =-\mathbf{Z}^{T} \mathcal{D} \mathbf{Z}
\end{aligned}
$$

Since $\mathbf{Y}^{T} \mathbf{X}=\mathbf{X}^{T} \mathbf{Y}$, it follows that

$$
\mathbf{X}^{T} \mathbf{A}^{T} \mathbf{Y}+\mathbf{Y}^{T} \mathbf{A} \mathbf{X}=-\left(\mathbf{Z}^{T} \mathbf{C X}+\mathbf{Z}^{T} \mathbf{B}^{T} \mathbf{Y}\right)^{T}=\mathbf{Z}^{T} \mathcal{D} \mathbf{Z}
$$

to establish equations (2.3) and (2.4).

Suppose now that $\mathbf{X q}=\mathbf{0}$, with $\mathbf{q} \neq \mathbf{0}$. From (2.3), it follows that $\mathbf{q}^{T} \mathbf{Z}^{T} \mathcal{D} \mathbf{Z} \mathbf{q}=0$ which in turn implies that $\mathbf{Z q}=\mathbf{0}$ since $\mathcal{D}$ is positive definite. Moreover, $\mathbf{Y q} \neq \mathbf{0}$ since $\left[\mathbf{0}, \mathbf{q}^{T} \mathbf{Y}^{T}, \mathbf{0},\right]=\mathbf{q}^{T}\left[\mathbf{X}^{T}, \mathbf{Y}^{T}, \mathbf{Z}^{T}\right]=(\mathbf{Q q})^{T}$, and $\mathbf{Q}$ is full rank. Thus, $\operatorname{Null}(\mathbf{X}) \cap N u l l(\mathbf{Y})=\{\mathbf{0}\}$ since $\mathbf{q}$ was arbitrarily chosen from $N u l l(\mathbf{X})$.

Now, equation (2.2) implies that

$$
\begin{aligned}
\mathbf{0} & =\mathbf{A X} \mathbf{q}+\mathbf{B Z} \mathbf{q}=\mathbf{X} \mathbf{R q} \\
\mathbf{A}^{T} \mathbf{Y q} & =\mathbf{A}^{T} \mathbf{Y} \mathbf{q}+\mathbf{C}^{T} \mathbf{Z} \mathbf{q}=-\mathbf{Y} \mathbf{R q} \\
\mathbf{B}^{T} \mathbf{Y q} & =\mathbf{C X} \mathbf{q}+\mathbf{B}^{T} \mathbf{Y} \mathbf{q}+\mathcal{D} \mathbf{Z} \mathbf{q}=\mathbf{0}
\end{aligned}
$$

Therefore, $\mathbf{R q}=\mathbf{N t}$ where $\mathbf{N}$ is full rank and $\operatorname{Null}(\mathbf{X})=\operatorname{Range}(\mathbf{N})$. Moreover,

$$
\mathbf{A}^{T} \mathbf{Y q}=-\mathbf{Y R q}=\mathbf{Y N}(-\mathbf{t}), \text { and } \mathbf{B}^{T} \mathbf{Y} \mathbf{q}=\mathbf{0} .
$$

Applying this argument successively to each column of $\mathbf{N}$ gives

$$
\begin{aligned}
\mathbf{R N} & =\mathbf{N T}, \\
\mathbf{A}^{T} \mathbf{Y N} & =\mathbf{Y N}(-\mathbf{T}), \\
\mathbf{B}^{T} \mathbf{Y N} & =\mathbf{0} .
\end{aligned}
$$

If $(-\mathbf{T}) \mathbf{y}=\mathbf{y} \lambda$ with $\mathbf{y} \neq \mathbf{0}$, put $\mathbf{v}=\mathbf{Y N y}$. Then $\mathbf{A}^{T} \mathbf{v}=\mathbf{v} \lambda$, and $\mathbf{B}^{T} \mathbf{v}=\mathbf{0}$, with $\mathbf{v} \neq \mathbf{0}$ since $N u l l(\mathbf{X}) \cap$ $\operatorname{Null}(\mathbf{Y})=\{\mathbf{0}\}$. Due to the Popov-Belevich-Hautus Lemma (Thm. 2.4.8 in [14]), this contradicts the assumption that $\Sigma$ is controllable.

A similar argument starting with $\mathbf{q} \in \operatorname{Null}(\mathbf{Y})$ will lead to a contradiction of the assumption that $\boldsymbol{\Sigma}$ is observable and this concludes the proof.

With these observations in hand, consider the following construction of $\mathbf{V}$ and $\mathbf{W}$. First, find a basis for an invariant subspace with all eigenvalues of $\mathbf{R}$ in the open right half-plane. Let $\mathbf{Q}_{x} \mathbf{S}^{2} \mathbf{Q}_{y}^{T}=\mathbf{X}^{T} \mathbf{Y}$ be the SVD of 
$\mathbf{X}^{T} \mathbf{Y}$ and note that $\mathbf{Q}_{y}=\mathbf{Q}_{x} \mathbf{J}$ where $\mathbf{J}$ is a signature matrix by virtue of the fact that $\mathbf{X}^{T} \mathbf{Y}$ is symmetric. If $\mathbf{S} \geq \mathbf{0}$ is nonsingular, put

$$
\mathbf{V}=\mathbf{X Q}_{x} \mathbf{S}^{-1}, \quad \mathbf{W}=\mathbf{Y Q}_{y} \mathbf{S}^{-1},
$$

so that $\mathbf{W}^{T} \mathbf{V}=\mathbf{I}$. Now, let $\hat{\mathbf{X}}:=\mathbf{S} \mathbf{Q}_{x}^{T}$ and $\hat{\mathbf{Y}}:=\mathbf{S} \mathbf{Q}_{y}^{T}$ and define

$$
\mathcal{V}:=\left[\begin{array}{rrr}
\mathbf{V} & \mathbf{0} & \mathbf{0} \\
\mathbf{0} & \mathbf{W} & \mathbf{0} \\
\mathbf{0} & \mathbf{0} & \mathbf{I}
\end{array}\right], \text { and } \mathcal{W}:=\left[\begin{array}{rrr}
\mathbf{W} & \mathbf{0} & \mathbf{0} \\
\mathbf{0} & \mathbf{V} & \mathbf{0} \\
\mathbf{0} & \mathbf{0} & \mathbf{I}
\end{array}\right]
$$

Observe that $\mathcal{W}^{T} \mathcal{V}=\mathbf{I}$ and that

$$
\left[\begin{array}{l}
\mathbf{X} \\
\mathbf{Y} \\
\mathbf{Z}
\end{array}\right]=\left[\begin{array}{rrr}
\mathbf{V} & \mathbf{0} & \mathbf{0} \\
\mathbf{0} & \mathbf{W} & \mathbf{0} \\
\mathbf{0} & \mathbf{0} & \mathbf{I}
\end{array}\right]\left[\begin{array}{l}
\hat{\mathbf{X}} \\
\hat{\mathbf{Y}} \\
\mathbf{Z}
\end{array}\right] \text {, and }\left[\begin{array}{l}
\mathbf{X} \\
\mathbf{Y} \\
\mathbf{0}
\end{array}\right]=\left[\begin{array}{rrr}
\mathbf{V} & \mathbf{0} & \mathbf{0} \\
\mathbf{0} & \mathbf{W} & \mathbf{0} \\
\mathbf{0} & \mathbf{0} & \mathbf{I}
\end{array}\right]\left[\begin{array}{c}
\hat{\mathbf{X}} \\
\hat{\mathbf{Y}} \\
\mathbf{0}
\end{array}\right]
$$

Therefore,

$$
\hat{\mathcal{A}}:=\mathcal{W}^{T} \mathcal{A} \mathcal{V}=\left[\begin{array}{ccc}
\hat{\mathbf{A}} & & \hat{\mathbf{B}} \\
& -\hat{\mathbf{A}}^{T} & -\hat{\mathbf{C}}^{T} \\
\hat{\mathbf{C}} & \hat{\mathbf{B}}^{T} & \mathbf{D}+\mathbf{D}^{T}
\end{array}\right]
$$

and

$$
\left[\begin{array}{ccc}
\hat{\mathbf{A}} & & \hat{\mathbf{B}} \\
& -\hat{\mathbf{A}}^{T} & -\hat{\mathbf{C}}^{T} \\
\hat{\mathbf{C}} & \hat{\mathbf{B}}^{T} & \mathbf{D}+\mathbf{D}^{T}
\end{array}\right]\left[\begin{array}{c}
\hat{\mathbf{X}} \\
\hat{\mathbf{Y}} \\
\mathbf{Z}
\end{array}\right]=\left[\begin{array}{c}
\hat{\mathbf{X}} \\
\hat{\mathbf{Y}} \\
\mathbf{0}
\end{array}\right] \mathbf{R}
$$

This shows that the spectral zeros $\mathcal{S}_{\hat{\mathrm{G}}}$ are a subset of the spectral zeros $\mathcal{S}_{\mathbf{G}}$ of the original system. Moreover, since $\mathcal{S}_{\hat{\mathbf{G}}}=\sigma(\mathbf{R}) \cup \sigma\left(-\mathbf{R}^{T}\right)$ and $\sigma(\mathbf{R})$ is in the open right half-plane, the reduced model has no spectral zeros on the imaginary axis.

It turns out that this construction gives a reduced model $\hat{\boldsymbol{\Sigma}}=(\hat{\mathbf{A}}, \hat{\mathbf{B}}, \hat{\mathbf{C}}, \mathbf{D})$ that is stable and passive. From the previous remarks, this will actually imply that the reduced system is strictly positive real. One could just apply Theorem 2.1 to establish this. However, it is instructive to prove passivity and stability directly from the construction. This will be established with the following results.

First, it is useful to note that $\hat{\mathbf{Y}} \hat{\mathbf{X}}^{-1}=\mathbf{S Q}_{y}^{T} \mathbf{Q}_{x} \mathbf{S}^{-1}=\mathbf{J}$, since $\mathbf{Q}_{y}=\mathbf{Q}_{x} \mathbf{J}$. Thus, $\mathbf{V}=\mathbf{X} \hat{\mathbf{X}}^{-1}$ and $\mathbf{W} \mathbf{J}=\mathbf{Y} \hat{\mathbf{X}}^{-1}$.

Lemma 2.3 The reduced model $\hat{\boldsymbol{\Sigma}}$ satisfies

$$
\begin{aligned}
\hat{\mathbf{A}}^{T}(-\mathbf{J})+(-\mathbf{J}) \hat{\mathbf{A}} & =-\mathbf{C}_{o}^{T} \mathbf{C}_{o} \\
\hat{\mathbf{B}}^{T}(-\mathbf{J})+\mathbf{W}_{o}^{T} \mathbf{C}_{o} & =\hat{\mathbf{C}} \\
\mathbf{D}+\mathbf{D}^{T} & =\mathbf{W}_{o}^{T} \mathbf{W}_{o}
\end{aligned}
$$

where $\mathbf{C}_{o}:=-\mathbf{W}_{o} \mathbf{Z} \hat{\mathbf{X}}^{-1}$.

Proof: From (2.3), it follows that

$$
\begin{aligned}
\hat{\mathbf{X}}^{-T} \mathbf{X}^{T} \mathbf{A}^{T} \mathbf{Y} \hat{\mathbf{X}}^{-1}+\hat{\mathbf{X}}^{-T} \mathbf{Y}^{T} \mathbf{A} \mathbf{X} \hat{\mathbf{X}}^{-1} & =\hat{\mathbf{X}}^{-T} \mathbf{Z}^{T} \mathcal{D} \mathbf{Z} \hat{\mathbf{X}}^{-1} \\
\mathbf{C X} \hat{\mathbf{X}}^{-1}+\mathbf{B}^{T} \mathbf{Y} \hat{\mathbf{X}}^{-1} & =-\mathcal{D} \mathbf{Z} \hat{\mathbf{X}}^{-1}
\end{aligned}
$$


and hence that

$$
\begin{aligned}
\mathbf{V}^{T} \mathbf{A}^{T} \mathbf{W} \mathbf{J}+\mathbf{J} \mathbf{W}^{T} \mathbf{A} \mathbf{V} & =\hat{\mathbf{X}}^{-T} \mathbf{Z}^{T} \mathcal{D} \mathbf{Z} \hat{\mathbf{X}}^{-1}=\mathbf{C}_{o}^{T} \mathbf{C}_{o} \\
\mathbf{C V}+\mathbf{B}^{T} \mathbf{W} \mathbf{J} & =-\mathcal{D} \mathbf{Z} \hat{\mathbf{X}}^{-1}=\mathbf{W}_{o}^{T} \mathbf{C}_{o},
\end{aligned}
$$

where $\mathbf{C}_{o}=-\mathbf{W}_{o} \mathbf{Z} \hat{\mathbf{X}}^{-1}$, since $\mathbf{D}+\mathbf{D}^{T}=\mathcal{D}=\mathbf{W}_{o}^{T} \mathbf{W}_{o}$. This gives the desired result.

Lemma 2.4 If $\mathbf{J}=-\mathbf{I}$ in Lemma (2.3), then $\mathbf{G}$ is positive real and the reduced order system $\hat{\mathbf{\Sigma}}$ is stable and passive.

Proof: Assume that $\mathbf{J}=-\mathbf{I}$ and that $\hat{\mathbf{A}} \mathbf{q}=\mathbf{q} \lambda$ with $\mathbf{q} \neq \mathbf{0}$. Then

$$
2 R e(\lambda)=\mathbf{q}^{T} \hat{\mathbf{A}}^{T} \mathbf{q}+\mathbf{q}^{T} \hat{\mathbf{A}} \mathbf{q}=-\left\|\mathbf{C}_{o} \mathbf{q}\right\|^{2} \leq 0 .
$$

If $0=\left\|\mathbf{C}_{o} \mathbf{q}\right\|=\left\|\mathbf{W}_{o} \mathbf{Z}\left(\hat{\mathbf{X}}^{-1} \mathbf{q}\right)\right\|$ then $\mathbf{Z}\left(\hat{\mathbf{X}}^{-1} \mathbf{q}\right)=\mathbf{0}$, since $\mathbf{W}_{o}$ is nonsingular.

It follows from the first block-row of 2.6 that

$$
\mathbf{q} \lambda=\hat{\mathbf{A}} \mathbf{q}=(\hat{\mathbf{A}} \hat{\mathbf{X}}+\hat{\mathbf{B} Z})\left(\hat{\mathbf{X}}^{-1} \mathbf{q}\right)=\hat{\mathbf{X}} \mathbf{R} \hat{\mathbf{X}}^{-1} \mathbf{q}
$$

This would imply that $\lambda \in \sigma(\mathbf{R})$, and hence that $\operatorname{Re}(\lambda)>0$ which is a contradiction and hence the stability of $\hat{\mathbf{A}}$ is established. Now, all of the conditions of the Positive Real Lemma ([3] Chap. 5, or Theorem (13.25) in [20]) are satisfied and the reduced system must also be passive.

It remains to show that $\mathbf{J}=-\mathbf{I}$. To do this, it is sufficient to show that $\hat{\mathbf{X}}^{T} \hat{\mathbf{Y}}$ is negative definite. Surprisingly, this follows primarily from the stability of the original system $\boldsymbol{\Sigma}$. The passivity assumption enters only in a very subtle way.

Lemma 2.5 The symmetric matrix $\mathbf{X}^{T} \mathbf{Y}$ is negative semi-definite and, as a consequence, the matrix $\hat{\mathbf{X}}^{T} \hat{\mathbf{Y}}$ in Lemma (2.3) is is also symmetric and negative semi-definite. If, in addition, $\mathbf{G}$ is strictly positive real then both matrices are negative definite. In either case, $\mathbf{J}=-\mathbf{I}$.

Proof: Assume for the moment that $\mathbf{G}$ is strictly positive real, i.e. has no spectral zeros on the imaginary axis. Then, the spectral zeros $\mathcal{S}_{\mathbf{G}}$ must consist of $n$ roots in the open right half plane together with their reflections across the imaginary axis. Let $\tilde{\mathbf{Q}}:=\left[\mathbf{Q}, \mathbf{Q}_{2}\right]$ and let $\tilde{\mathbf{R}}:=\left[\begin{array}{cc}\mathbf{R} & \mathbf{R}_{12} \\ \mathbf{0} & \mathbf{R}_{22}\end{array}\right]$ represent an extension of the partial Schur decomposition defined by $\mathbf{Q}$ and $\mathbf{R}$ to include all of the spectral zeros in the open right half-plane as eigenvalues of $\tilde{\mathbf{R}}$ forming a new partial Schur decomposition of order $n$. Thus, $\mathcal{A} \tilde{\mathbf{Q}}=\mathcal{E} \tilde{\mathbf{Q}} \tilde{\mathbf{R}}$ and one can partition

$$
\tilde{\mathbf{Q}}=\left[\begin{array}{ll}
\mathbf{X} & \mathbf{X}_{2} \\
\mathbf{Y} & \mathbf{Y}_{2} \\
\mathbf{Z} & \mathbf{Z}_{2}
\end{array}\right]
$$

as before. Now apply Lemma (2.2) to this system with $\tilde{\mathbf{X}}:=\left[\mathbf{X}, \mathbf{X}_{2}\right]$ in place of $\mathbf{X}, \tilde{\mathbf{Y}}=\left[\mathbf{Y}, \mathbf{Y}_{2}\right]$ in place of $\mathbf{Y}$, $\tilde{\mathbf{Z}}:=\left[\mathbf{Z}, \mathbf{Z}_{2}\right]$ in place of $\mathbf{Z}$ and $\tilde{\mathbf{R}}$ in place of $\mathbf{R}$.

From equation (2.3),

$$
\tilde{\mathbf{X}}^{T} \mathbf{A}^{T} \tilde{\mathbf{Y}}+\tilde{\mathbf{Y}}^{T} \mathbf{A} \tilde{\mathbf{X}}=\tilde{\mathbf{Z}}^{T} \mathcal{D} \tilde{\mathbf{Z}} \text { with } \tilde{\mathbf{X}} \text { nonsingular. }
$$

Thus,

$$
\mathbf{A}^{T}\left(\tilde{\mathbf{Y}} \tilde{\mathbf{X}}^{-1}\right)+\left(\tilde{\mathbf{Y}} \tilde{\mathbf{X}}^{-1}\right)^{T} \mathbf{A}=\tilde{\mathbf{X}}^{-T} \tilde{\mathbf{Z}}^{T} \mathbf{W}_{o}^{T} \mathbf{W}_{o} \tilde{\mathbf{Z}} \tilde{\mathbf{X}}^{-1}
$$


Since Lemma (2.1) implies $\mathcal{Q}:=\tilde{\mathbf{Y}} \tilde{\mathbf{X}}^{-1}$ is symmetric, it follows that

$$
\mathbf{A}^{T} \mathcal{Q}+\mathcal{Q} \mathbf{A}=\tilde{\mathbf{C}}_{o}^{T} \tilde{\mathbf{C}}_{o}
$$

with $\tilde{\mathbf{C}}_{o}:=\mathbf{W}_{o} \tilde{\mathbf{Z}} \tilde{\mathbf{X}}^{-1}$. Thus, $\mathcal{Q}$ is negative semi-definite and it follows that $\tilde{\mathbf{X}}^{T} \tilde{\mathbf{Y}}=\tilde{\mathbf{X}}^{T} \mathcal{Q} \tilde{\mathbf{X}}$ is also symmetric negative semi-definite. However, since $\tilde{\mathbf{Y}}^{T} \tilde{\mathbf{X}}$ is nonsingular, this matrix is actually negative definite. Moreover, since $\mathbf{X}^{T} \mathbf{Y}$ is a leading principle sub-matrix of $\tilde{\mathbf{X}}^{T} \tilde{\mathbf{Y}}$, it must also be negative definite.

Thus, the desired result holds when $\mathbf{G}$ is strictly positive real. The strictly positive condition can be relaxed to positive real using a continuity argument based upon considering $\mathcal{D}_{\epsilon}:=\epsilon \mathbf{I}+\mathcal{D}$ for $\epsilon$ decreasing to 0 . In this limit, definiteness may be lost. Thus, $\mathbf{X}^{T} \mathbf{Y}$ is only negative semi-definite in general.

Now, since $\mathbf{Q}_{x} \mathbf{S}^{2} \mathbf{J} \mathbf{Q}_{x}^{T}=\hat{\mathbf{X}}^{T} \hat{\mathbf{Y}}=\hat{\mathbf{X}}^{T} \mathbf{V}^{T} \mathbf{W} \hat{\mathbf{Y}}=\mathbf{X}^{T} \mathbf{Y}$, it follows that the diagonal elements of $\mathbf{S}^{2} \mathbf{J}$ are non-positive. For every positive diagonal element of $\mathbf{S}$, the corresponding diagonal element of $\mathbf{J}$ is negative. The remaining diagonal elements of $\mathbf{J}$ are of arbitrary sign and thus may be taken as negative. Hence $\mathbf{J}=-\mathbf{I}$.

Note that the only use of passivity in this proof was the fact that an arbitrarily small positive perturbation to $\mathbf{D}$ would move all of the spectral zeros off of the imaginary axis. If $\mathbf{G}^{T}\left(-i \omega_{o}\right)+\mathbf{G}\left(i \omega_{o}\right)<0$ for some $\omega_{o} \in \mathbb{R}$, then this would be impossible and the construction would fail.

Dealing with degeneracy: If $\mathbf{S}$ is singular then equations (2.5) become invalid and the interpolation scheme will break down. However, it is still possible to construct a non-interpolatory passive and stable reduced model. Beginning with $\mathbf{X}, \mathbf{Y}$ from (2.2) and with the SVD $\mathbf{Q}_{x} \mathbf{S}^{2} \mathbf{Q}_{y}^{T}=\mathbf{X}^{T} \mathbf{Y}$, specify a cut off tolerance $\tau_{c} \in(0,1)$ and let $j$ be the largest positive integer such that

$$
\sigma_{j} \geq \tau_{c} \sigma_{1}, \text { where } \sigma_{j}:=\mathbf{S}(j, j) .
$$

Define $\mathbf{Q}_{j}:=\mathbf{Q}_{x}(:, 1: j), \mathbf{S}_{j}:=\mathbf{S}(1: j, 1: j)$, and then let $\mathbf{X}_{j}^{I}:=\mathbf{Q}_{j} \mathbf{S}_{j}^{-1}$. Replace $\hat{\mathbf{X}}^{-1}$ by $\mathbf{X}_{j}^{I}$ in equations (2.7) and (2.8). Then equations (2.9) and (2.10) are obtained with $\mathbf{V}:=\mathbf{X} \mathbf{X}_{j}^{I}, \mathbf{W}:=-\mathbf{Y} \mathbf{X}_{j}^{I}$ and with $\mathbf{C}_{o}:=$ $-\mathbf{W}_{o} \mathbf{Z X} \mathbf{X}_{j}^{I}$. From Lemma (2.5) it follows that $\mathbf{X}^{T} \mathbf{Y}$ is negative semi-definite regardless of $\mathbf{S}$ and hence that $\mathbf{Q}_{y}=-\mathbf{Q}_{x}$. Therefore, $\mathbf{W}^{T} \mathbf{V}=-\mathbf{S}_{j}^{-1} \mathbf{Q}_{j}^{T} \mathbf{Y}^{T} \mathbf{X} \mathbf{Q}_{j} \mathbf{S}_{j}^{-1}=-\mathbf{S}_{j}^{-1} \mathbf{Q}_{j}^{T} Q_{x}(-\mathbf{S}) \mathbf{Q}_{x}^{T} \mathbf{Q}_{j} \mathbf{S}_{j}^{-1}=\mathbf{S}_{j}^{-1} \mathbf{S}_{j}=\mathbf{I}$.

This derivation implies that Lemma (2.3) holds true and thus the reduced model obtained in this way must also be passive. However, it may not be stable. The relation (2.11) is still obtained but (2.12) is no longer valid. However, since $\mathbf{X}_{j}^{I}$ is full rank and $\mathbf{W}_{o}$ is nonsingular, the stability of the reduced model is obtained if $\mathbf{Z}$ is full rank. In any case, $\hat{\mathbf{A}}$ will have no eigenvalues in the right half-plane.

This discussion is valid for any index $j$ such that $\mathbf{S}_{j}$ is nonsingular. The interpolation conditions are lost but each such model is passive and also stable if $\mathbf{Z}$ is full rank.

\section{Riccati Equations, Minimal Solutions, and Balancing}

Another approach to model reduction of passive systems is based upon the solution of a certain pair of Riccati equations. There are a number of solution methods for algebraic Riccati equations that involve numerical computation of a stable invariant subspace of a related Hamiltonian matrix. These include the Schur vector method of Laub [15], the Hamiltonian QR-algorithm of Byers [8], the HHDR-algorithm of Bunse-Gerstner and Mehrmann [7], and the matrix sign function method [9] and the multi-shift method of Ammar, Benner, and Mehrmann [1].

The approach just described for passive reduction is indeed related, and in fact, the associated pair of Riccati equations is implicitly solved when the invariant subspace basis has been computed. The following derivation of these relations is offered as an interesting by-product of the passive reduction scheme. This connection may lead to an algorithmic consequence, but that possibility will be left to future research. To begin the discussion, it shall be assumed that the transfer function $\mathbf{G}(s)$ is strictly positive real. Initially, in Equation (2.2) consider the invariant 
subspace corresponding to the $n$ spectral zeros in the open right half-plane. Thus, $\mathbf{X}, \mathbf{Y}$ are both nonsingular and $\mathbf{X}^{T} \mathbf{Y}$ is symmetric and negative semi-definite.

From Equations (2.3) and (2.4) one obtains

$$
\begin{aligned}
\mathbf{A}^{T} \mathcal{Q}+\mathcal{Q} \mathbf{A} & =-\mathbf{C}_{o}^{T} \mathbf{C}_{o}, \quad \mathbf{C}-\mathbf{B}^{T} \mathcal{Q}=-\mathbf{W}_{o}^{T} \mathbf{C}_{o} \\
\mathbf{A} \mathcal{P}+\mathcal{P} \mathbf{A}^{T} & =-\mathbf{B}_{o} \mathbf{B}_{o}^{T}, \quad \mathbf{B}^{T}-\mathbf{C} \mathcal{P}=-\mathbf{W}_{o}^{T} \mathbf{B}_{o}^{T}
\end{aligned}
$$

with

$$
\begin{array}{ll}
\mathcal{Q}=-\mathbf{Y} \mathbf{X}^{-1}, & \mathbf{C}_{o}=\mathbf{W}_{o} \mathbf{Z} \mathbf{X}^{-1} \\
\mathcal{P}=\mathcal{Q}^{-1}, & \mathbf{B}_{o}^{T}=\mathbf{W}_{o} \mathbf{Z} \mathbf{Y}^{-1}
\end{array}
$$

Since $\mathbf{W}_{o}$ nonsingular,

$$
\mathbf{C}_{o}=-\mathbf{W}_{o}^{-T}\left(\mathbf{C}-\mathbf{B}^{T} \mathcal{Q}\right), \text { and } \mathbf{B}_{o}^{T}=-\mathbf{W}_{o}^{-T}\left(\mathbf{B}^{T}-\mathbf{C} \mathcal{P}\right) .
$$

Substituting these expressions for $\mathbf{C}_{o}, \mathbf{B}_{o}$ into equations (3.1) and (3.2) gives the following two Riccati equations

$$
\begin{aligned}
\mathbf{A}^{T} \mathcal{Q}+\mathcal{Q} \mathbf{A} & =-\left(\mathbf{C}^{T}-\mathcal{Q} \mathbf{B}\right) \mathcal{D}^{-1}\left(\mathbf{C}-\mathbf{B}^{T} \mathcal{Q}\right) \\
\mathbf{A} \mathcal{P}+\mathcal{P} \mathbf{A}^{T} & =-\left(\mathbf{B}-\mathcal{P} \mathbf{C}^{T}\right) \mathcal{D}^{-1}\left(\mathbf{B}^{T}-\mathbf{C} \mathcal{P}\right)
\end{aligned}
$$

Since $\mathbf{Y} \mathbf{X}^{-1}=\mathbf{X}^{-T}\left(\mathbf{X}^{T} \mathbf{Y}\right) \mathbf{X}^{-1}$ and $\mathbf{X}^{T} \mathbf{Y}$ is symmetric negative definite, it follows that both $\mathcal{P}$ and $\mathcal{Q}$ are positive definite. These are the equations one normally solves. It is well known that these equations do not have unique solutions, and usually it is desirable to have either $\mathcal{Q}$ or $\mathcal{P}$ be a minimal solution.

Definition: $\mathcal{Q}$ is a maximal solution if $\mathcal{Q} \geq \hat{\mathcal{Q}}$ for any other solution to (3.3) and $\mathcal{Q}$ is a minimal solution if $\mathcal{Q} \leq \hat{\mathcal{Q}}$ for any other solution to (3.3). Likewise, $\mathcal{P}$ is a maximal solution if $\mathcal{P} \geq \hat{\mathcal{P}}$ for any other solution to (3.4) and $\mathcal{P}$ is a minimal solution if $\mathcal{P} \leq \hat{\mathcal{P}}$ for any other solution to (3.4).

Since $\mathcal{P}=\mathcal{Q}^{-1}$, is clear that $\mathcal{Q}$ is a maximal solution to (3.3) if and only if $\mathcal{P}$ is minimal for (3.4). A natural question then is to ask which solution pair is constructed by this method?

Theorem 3.1 The solution $\mathcal{Q}$ to (3.3) constructed above is maximal and hence $\mathcal{P}$ is a minimal solution to (3.4).

Proof: Let $\hat{\mathcal{Q}}=\mathcal{Q}+\mathbf{E}$ be any other symmetric solution to (3.3). To show $\hat{\mathcal{Q}} \leq \mathcal{Q}$ it is sufficient to show that $\mathbf{E}$ is negative semi-definite. To this end, beginning with

$$
\begin{aligned}
& \mathbf{A}^{T} \mathcal{Q}+\mathcal{Q} \mathbf{A}=-\left(\mathbf{C}^{T}-\mathcal{Q} \mathbf{B}\right) \mathcal{D}^{-1}\left(\mathbf{C}-\mathbf{B}^{T} \mathcal{Q}\right) \\
& \mathbf{A}^{T} \hat{\mathcal{Q}}+\hat{\mathcal{Q}} \mathbf{A}=-\left(\mathbf{C}^{T}-\hat{\mathcal{Q}} \mathbf{B}\right) \mathcal{D}^{-1}\left(\mathbf{C}-\mathbf{B}^{T} \hat{\mathcal{Q}}\right)
\end{aligned}
$$

and expanding the second equation for $\hat{\mathcal{Q}}=\mathcal{Q}+\mathbf{E}$ about $\mathcal{Q}$ gives the following equation for the perturbation $\mathbf{E}$.

$$
\mathbf{A}^{T} \mathbf{E}+\mathbf{E} \mathbf{A}=\mathbf{E B} \mathcal{D}^{-1}\left(\mathbf{C}-\mathbf{B}^{T} \mathcal{Q}\right)+\left(\mathbf{C}^{T}-\mathcal{Q B}\right) \mathcal{D}^{-1} \mathbf{B}^{T} \mathbf{E}-\mathbf{E B} \mathcal{D}^{-1} \mathbf{B}^{T} \mathbf{E} .
$$

Collecting terms yields the equation

$$
\tilde{\mathbf{A}}^{T} \mathbf{E}+\mathbf{E} \tilde{\mathbf{A}}+\mathbf{E B} \mathcal{D}^{-1} \mathbf{B}^{T} \mathbf{E}=\mathbf{0} \text {, with } \tilde{\mathbf{A}}:=\mathbf{A}-\mathbf{B} \mathcal{D}^{-1}\left(\mathbf{C}-\mathbf{B}^{T} \mathcal{Q}\right) .
$$


Notice that the matrix $\tilde{\mathbf{A}}$ is anti-stable (all eigenvalues in the open right half-plane) since

$$
\begin{aligned}
\tilde{\mathbf{A}} & =\left[\mathbf{A X}-\mathbf{B} \mathcal{D}^{-1}\left(\mathbf{C X}-\mathbf{B}^{T} \mathcal{Q} \mathbf{X}\right)\right] \mathbf{X}^{-1} \\
& =\left[\mathbf{A X}-\mathbf{B} \mathcal{D}^{-1}\left(\mathbf{C X}+\mathbf{B}^{T} \mathbf{Y}\right)\right] \mathbf{X}^{-1} \\
& =\left[\mathbf{A X}-\mathbf{B} \mathcal{D}^{-1}(-\mathcal{D} \mathbf{Z})\right] \mathbf{X}^{-1} \\
& =[\mathbf{A X}+\mathbf{B Z}] \mathbf{X}^{-1} \\
& =\mathbf{X R X}^{-1}
\end{aligned}
$$

This shows that the eigenvalues of $\tilde{\mathbf{A}}$ are, in fact, the $n$ spectral zeros in the open right half-plane. Since $\mathbf{E B} \mathcal{D}^{-1} \mathbf{B}^{T} \mathbf{E}$ is positive semi-definite, it follows that $\mathbf{E}$ must be negative semi-definite. Hence, $\hat{\mathcal{Q}} \leq \mathcal{Q}$ as claimed.

To get the minimal solution, instead of interpolating the spectral zeros in the right half-plane, one should interpolate the spectral zeros in the open left half-plane. To see this, note that all results of the previous section are valid when the matrix $\mathbf{R}$ has the spectral zeros in the open left half-plane as its eigenvalues. The only change needed in the proofs comes at equation (2.12) in the proof of Lemma (2.4). Instead of a contradiction, one gets $\lambda \in \sigma(\mathbf{R})$ which implies $\operatorname{Re}(\lambda)<0$ directly when $\mathbf{Z} \hat{\mathbf{X}}^{-1} \mathbf{q}=\mathbf{0}$. Now, an argument following the proof of Theorem 3.1 will give $\mathbf{E}$ positive semidefinite since $\tilde{\mathbf{A}}=\mathbf{X R X} \mathbf{X}^{-1}$ will show it to be stable. Thus, the following theorem holds.

Theorem 3.2 If a solution $\mathcal{Q}_{-}$to (3.3) is constructed as above but with $\mathbf{R}$ having the spectral zeros of $\mathbf{G}(s)$ in the open left half-plane as its eigenvalues, then $\mathcal{Q}_{-}$is minimal and hence $\mathcal{P}_{+}=\mathcal{Q}_{-}^{-1}$ is a maximal solution to (3.4).

These results indicate how a balanced reduction can be constructed that will also be passive. Construct a minimal solution $\mathcal{P}_{-}$to 3.4 by interpolating the spectral zeros in the open right half-plane and a minimal solution $\mathcal{Q}_{-}$by interpolating zeros in the open right half-plane. Then compute a balancing transformation from the SVD of $\left(\mathcal{P}_{-}\right)\left(\mathcal{Q}_{-}\right)$(or better from the product of the Cholesky factors).

Reduced Order Balancing It is also possible to produce a reduced order approximate balancing transformation without first balancing the full system. The alternative is to perform the procedure just as described in Section 2 to obtain a reduced model from $\mathbf{X}, \mathbf{Y}, \hat{\mathbf{X}}, \hat{\mathbf{Y}}$ corresponding to interpolation of a selected set of spectral zeros in the open right half-plane (a specific selection criterion is given in the next section). The conditions of Lemma (2.3) are satisfied along with equation (2.6) and with $\hat{\mathbf{Y}} \hat{\mathbf{X}}^{-1}=\mathbf{J}=-\mathbf{I}$. Recall that the reduced model is passive, stable and that there are no spectral zeros on the imaginary axis. Moreover, there are two associated reduced Riccati equations

$$
\begin{aligned}
\hat{\mathbf{A}}^{T} \hat{\mathcal{Q}}+\hat{\mathcal{Q}} \hat{\mathbf{A}} & =-\left(\hat{\mathbf{C}}^{T}-\hat{\mathcal{Q}} \hat{\mathbf{B}}\right) \mathcal{D}^{-1}\left(\hat{\mathbf{C}}-\hat{\mathbf{B}}^{T} \hat{\mathcal{Q}}\right) \\
\hat{\mathbf{A}} \hat{\mathcal{P}}+\hat{\mathcal{P}} \hat{\mathbf{A}}^{T} & =-\left(\hat{\mathbf{B}}-\hat{\mathcal{P}} \hat{\mathbf{C}}^{T}\right) \mathcal{D}^{-1}\left(\hat{\mathbf{B}}^{T}-\hat{\mathbf{C}} \hat{\mathcal{P}}\right) .
\end{aligned}
$$

and Theorem 3.1 implies $\hat{\mathcal{P}}=\hat{\mathcal{Q}}^{-1}$ is minimal for (3.6) with $\hat{\mathcal{Q}}:=-\hat{\mathbf{Y}} \hat{\mathbf{X}}^{-1}=\mathbf{I}$. The plan is to balance the reduced system and to do this, it is only necessary to compute a basis for the invariant subspace of the reduced system corresponding to the reduced spectral zeros in the open left half-plane. That is, compute $\tilde{\mathbf{X}}, \tilde{\mathbf{Y}}, \tilde{\mathbf{Z}}$ such that

$$
\left[\begin{array}{ccc}
\hat{\mathbf{A}} & & \hat{\mathbf{B}} \\
& -\hat{\mathbf{A}}^{T} & -\hat{\mathbf{C}}^{T} \\
\hat{\mathbf{C}} & \hat{\mathbf{B}}^{T} & \mathbf{D}+\mathbf{D}^{T}
\end{array}\right]\left[\begin{array}{c}
\tilde{\mathbf{X}} \\
\tilde{\mathbf{Y}} \\
\tilde{\mathbf{Z}}
\end{array}\right]=\left[\begin{array}{c}
\tilde{\mathbf{X}} \\
\tilde{\mathbf{Y}} \\
\mathbf{0}
\end{array}\right] \tilde{\mathbf{R}}
$$

is a partial real Schur decomposition of the reduced system and the eigenvalues of $\tilde{\mathbf{R}}$ are the spectral zeros of 
the reduced model in the open left half-plane (consisting of $\sigma\left(-\mathbf{R}^{T}\right)$ ). Now, construct the minimal solution $\tilde{\mathcal{Q}}$ to (3.5) as $\tilde{\mathcal{Q}}=\tilde{\mathbf{Y}} \tilde{\mathbf{X}}^{-1}$. The balancing transformation for the reduced model is then constructed from the SVD of $\hat{\mathcal{P}} \tilde{\mathcal{Q}}=\tilde{\mathcal{Q}}$.

Let $\tilde{\mathcal{Q}}=\mathbf{U} \mathcal{S}^{2} \mathbf{U}^{T}$ be the SVD of $\tilde{\mathcal{Q}}$. Note that

$$
\tilde{\mathcal{Q}}\left(\mathbf{U} \mathcal{S}^{-1 / 2}\right)=\left(\mathbf{U} \mathcal{S}^{1 / 2}\right) \mathcal{S}, \text { and } \hat{\mathcal{P}}\left(\mathbf{U} \mathcal{S}^{1 / 2}\right)=\left(\mathbf{U} \mathcal{S}^{-1 / 2}\right) \mathcal{S}
$$

and also note that singularity of $\mathcal{S}$ can be dealt with by truncation as before. Let

$$
\hat{\mathbf{A}}_{\beta}:=\mathcal{S}^{1 / 2} \mathbf{U}^{T} \hat{\mathbf{A}} \mathbf{U S} \mathcal{S}^{-1 / 2}, \hat{\mathbf{B}}_{\beta}:=\mathcal{S}^{1 / 2} \mathbf{U} \hat{\mathbf{B}}, \hat{\mathbf{C}}_{\beta}:=\hat{\mathbf{C}} \mathbf{U} \mathcal{S}^{-1 / 2} .
$$

Now, $\tilde{\mathcal{Q}}$ is a minimal solution to equation (3.5) and hence

$$
\hat{\mathbf{A}}^{T} \tilde{\mathcal{Q}}+\tilde{\mathcal{Q}} \hat{\mathbf{A}}=-\left(\hat{\mathbf{C}}^{T}-\tilde{\mathcal{Q}} \hat{\mathbf{B}}\right) \mathcal{D}^{-1}\left(\hat{\mathbf{C}}-\hat{\mathbf{B}}^{T} \tilde{\mathcal{Q}}\right)
$$

Multiplying this equation on the left by $\mathcal{S}^{-1 / 2} \mathbf{U}^{T}$ and on the right by $\mathbf{U} \mathcal{S}^{-1 / 2}$ and then applying relations (3.7) and (3.8) gives

$$
\hat{\mathbf{A}}_{\beta}^{T} \mathcal{S}+\mathcal{S} \hat{\mathbf{A}}_{\beta}=-\left(\hat{\mathbf{C}}_{\beta}^{T}-\mathcal{S} \hat{\mathbf{B}}_{\beta}\right) \mathcal{D}^{-1}\left(\hat{\mathbf{C}}_{\beta}-\hat{\mathbf{B}}_{\beta}^{T} \mathcal{S}\right)
$$

On the other hand, multiplying equation (3.6) the left by $\mathcal{S}^{1 / 2} \mathbf{U}^{T}$ and on the right by $\mathbf{U} \mathcal{S}^{1 / 2}$ and then applying relations (3.7) and (3.8) gives

$$
\hat{\mathbf{A}}_{\beta} \mathcal{S}+\mathcal{S} \hat{\mathbf{A}}_{\beta}^{T}=-\left(\hat{\mathbf{B}}_{\beta}-\mathcal{S} \hat{\mathbf{C}}_{\beta}^{T}\right) \mathcal{D}^{-1}\left(\hat{\mathbf{B}}_{\beta}^{T}-\hat{\mathbf{C}}_{\beta} \mathcal{S}\right) .
$$

Thus, the reduced model $\hat{\boldsymbol{\Sigma}}_{\beta}:=\left(\hat{\mathbf{A}}_{\beta}, \hat{\mathbf{B}}_{\beta}, \hat{\mathbf{C}}_{\beta}, \mathbf{D}\right)$ is balanced, stable, and passive with a strictly positive real transfer function $\hat{\mathbf{G}}_{\beta}(s)$. Moreover, the dimension of this model can be further reduced by simple truncation (deleting the smallest singular values first) while retaining these properties. Finally, note that this may be done even when the initial reduced model is non-interpolatory. Thus, a balanced reduced model may be obtained even in the face of degeneracy.

If this procedure is carried out with $k=n$, i.e., with the full system, then there is an error bound available due to S. Gugercin [12] on the $\mathcal{H}_{\infty}$ norm of the error. However, no error bound is yet available if the system is first reduced to size $k<n$ and then balanced as just described.

\section{Algorithms for Passivity Preserving Reduced Models}

The results of the previous section establish the passivity of $\hat{\boldsymbol{\Sigma}}$ and, in addition, they establish that $\mathcal{S}_{\hat{\mathbf{G}}} \subset \mathcal{S}_{\mathbf{G}}$ corresponding to the spectral zeros appearing as eigenvalues of $\mathbf{R}$ in (2.2). This leads to the following algorithm.

In the algorithm shown in Figure 1, it is assumed that $\mathcal{A}$ and $\mathcal{E}$ represent the blocked matrices defined by (2.1). For small to medium scale dense problems, these matrices might actually be formed and then the desired partial Schur decompostion would be extracted from the full eigensystem. For large sparse problems, this would be impractical and inefficient. The algorithm as posed is appropriate for real matrices, and in particular, all arithmetic stays real throughout. A partial real Schur decomposition is approproate since it will automatically keep complex congugate pairs of spectral zeros together. The parameter $k$ that specifies the order of the reduced model will perhaps need to be adjusted by 1 to accomodate this.

For large scale problems, an implicitly restarted Arnoldi (IRA) [19] method would be quite suitable. It naturally produces a partial real Schur form corresponding to a desired set of eigenvalues (spectral zeros here). One could use eigs in Matlab or ARPACK in Fortran [16] to find such an invariant subspace. One choice for selecting the 


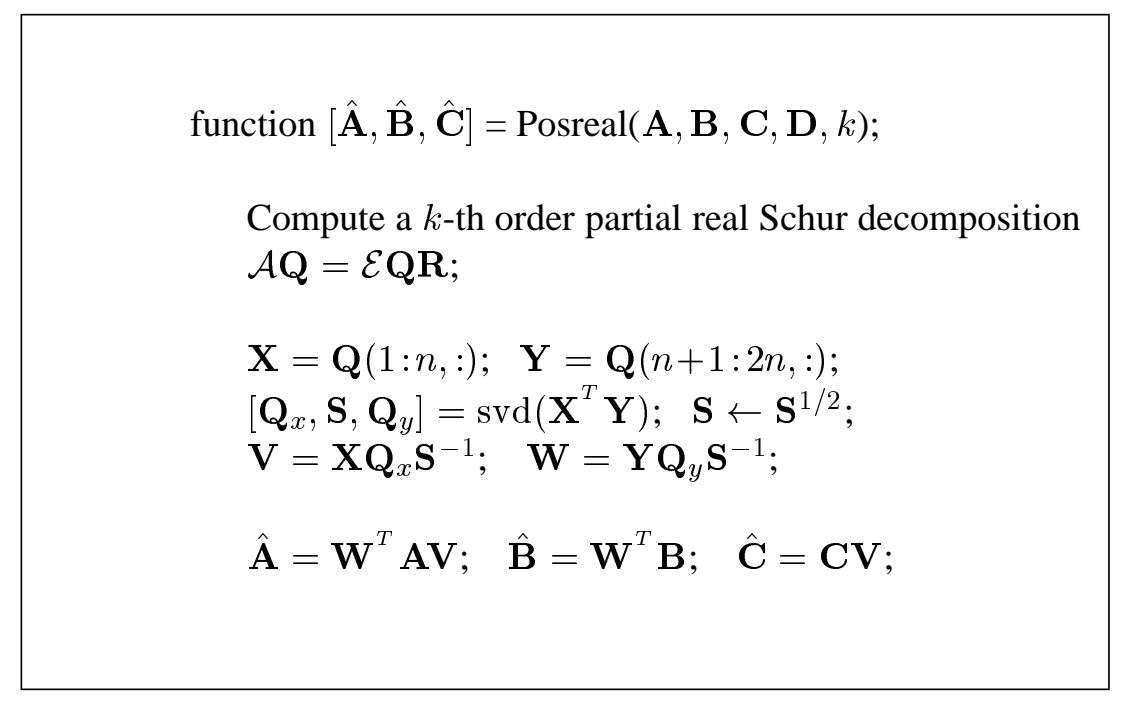

Figure 1: Positive Real Model Reduction

spectral zeros might be to compute the $k$ eigenvalues of largest real part. However, there is another choice that seems quite natural and which works well with an IRA method.

A convenient spectral transformation is obtained with the Cayley transformation

$$
\mathcal{C}_{\mu}:=(\mu \mathcal{E}-\mathcal{A})^{-1}(\mu \mathcal{E}+\mathcal{A})
$$

where $\mu \geq 0$ is a real shift. With a proper choice of $\mu$, this will provide for rapid convergence to an invariant subspace corresponding to $k$ transformed eigenvalues of largest magnitude:

$$
(\mu \mathcal{E}-\mathcal{A})^{-1}(\mu \mathcal{E}+\mathcal{A}) \mathbf{Q}=\mathbf{Q} \hat{\mathbf{R}}
$$

so that

$$
\mathcal{A} \mathbf{Q}=\mathcal{E} \mathbf{Q R}, \text { where } \mathbf{R}:=\mu(\hat{\mathbf{R}}-\mathbf{I})(\hat{\mathbf{R}}+\mathbf{I})^{-1} .
$$

This gives the partial Schur decomposition as required by the algorithm in Figure 1. The implementation will require two sparse direct factorizations of $\mathbf{A}-\mu \mathbf{I}$ and $\mathbf{A}+\mu \mathbf{I}$. The Cayley transformation $\mathcal{C}_{\mu}$ may then be applied to an arbitrary vector using a blocked matrix-vector product followed by a blocked Gaussian elimination. Interestingly enough, computing the $k$ eigenvalues of largest magnitude for this Cayley transformation is related to computing $k$ eigenvalues of largest real part for the original pair $(\mathcal{A}, \mathcal{E})$ in a very special way. A circle of radius $\rho>1$ centered at the origin is the image of a circle $\mathcal{D}_{\rho}$ of radius $\frac{2 \mu \rho}{\rho^{2}-1}$ centered at $\mu \frac{\rho^{2}+1}{\rho^{2}-1}$. If $\rho$ is the radius of the circle centered at the origin drawn through the selected eigenvalue(s) of smallest magnitude, then the $k$ selected eigenvalues are images of the spectral zeros interior to the circle $\mathcal{D}_{\rho}$ (shown in Figure 2) and this gives the interpolation points. As $\rho \rightarrow 1$ the interior circle $\mathcal{D}_{\rho}$ tends to include all of the right half-plane.

Example. The following graphs show the result of applying this scheme to an RLC circuit of order 201. The circuit is an RLC ladder network. The state variables are as follows: $x_{1}$, the voltage across $C_{1} ; x_{2}$, the current through $L_{1} ; x_{3}$, the voltage across $C_{2} ; x_{4}$, the current through $L_{2}$; and $x_{5}$, the voltage across $C_{3}$, etc. In general, $n$ is odd and $x_{2 i-1}$ is the voltage across capacitor $C_{i}$ for $i=1,2, \ldots \frac{n+1}{2}$, while $x_{2 i}$ is the current through inductor $L_{i}$ for $i=1,2, \ldots \frac{n-1}{2}$. There are two resistors $R_{1}, R_{2}$ placed at either end of the "ladder" as shown in Figure 3 for an order $n=5$ example.

The input is the voltage $u$ and the output is the current $y$ as shown in Figure 3. It is assumed that all the capacitors and inductors have unit value, while $R_{1}=\frac{1}{2}, R_{2}=\frac{1}{5}$. A minimal realization for the order $n=5$ 

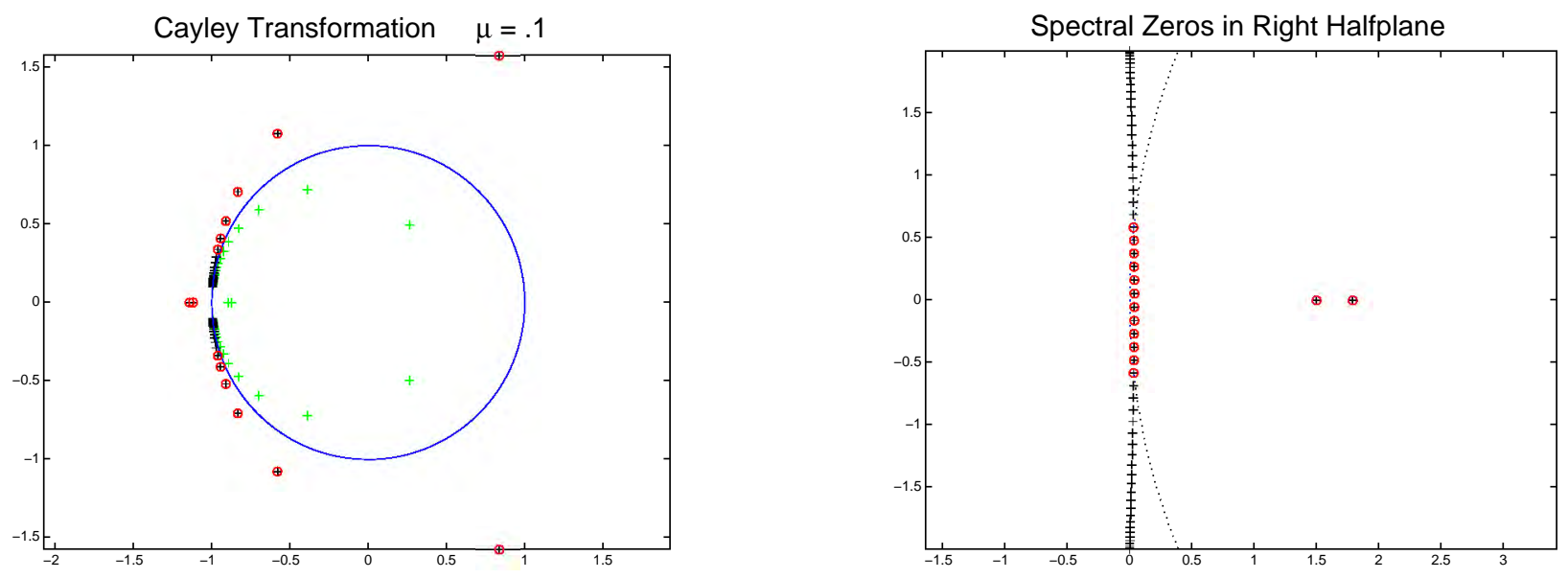

Figure 2: The Cayley transformed spectral zeros (+) and interpolation points selected (o) (reduced order spectral zeros).

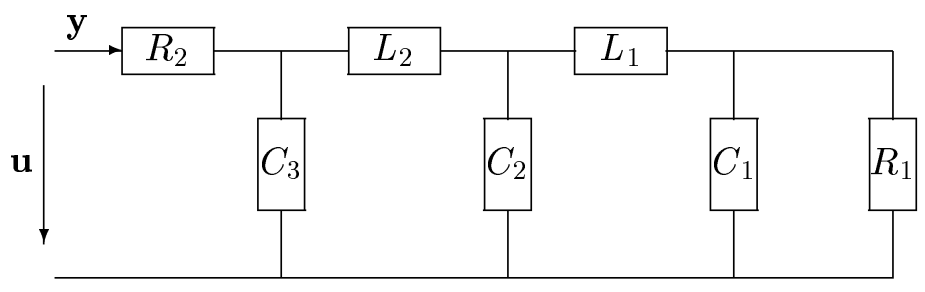

Figure 3: RLC circuit of order 5

example is:

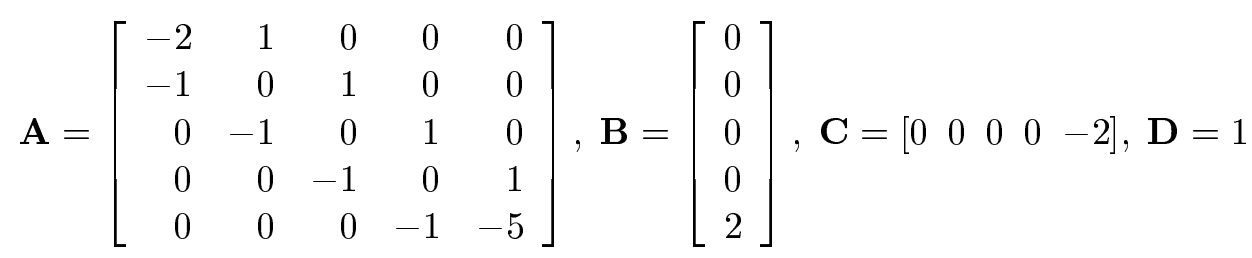

For general $n$, the matrix $\mathbf{A}$ is simply extended with value 1 on the super diagonal, the value -1 on the sub-diagonal and with the values -2 , and -5 in the $(1,1)$ and $(n, n)$ positions. The vectors $\mathbf{B}, \mathbf{C}$ are extended in the obvious way by introducing zero entries in the first $n-1$ positions.

The passive reduction scheme was applied to an RLC ladder system of order $n=201$ using an IRA method with the Cayley transformation (shift $\mu=.1$ ). A reduced model of order 20 was constructed. The graphs in Figure 4 and Figure 5 illustrate the effectiveness of the procedure. The distribution of the spectral zeros are similar to those shown in Figure 2 for a smaller problem. That figure shows the effect of the transformation and the interpolation points.

To explore the balancing procedure, a dense method was applied to attempt to interpolate all of the spectral zeros in the open right half-plane. It turns out that this resulted a degeneracy since $\sigma_{n}=S(n, n)$ was very small in magnitude relative to the first $n-1$ singular values. The procedure described for treating degeneracy in the previous section was used. Then the balancing scheme given by equations (3.8) and (3.7) was applied to the same RLC ladder system of order $n=201$. The (positive real) singular values $\mathcal{S}$ did not decay at all. Nevertheless, a balanced reduced model of order 10 was constructed by the usual truncation. The results are remarkable even though there was very little decay in the singular values. The graphs in Figure 6 and Figure 7 illustrate the excellent 

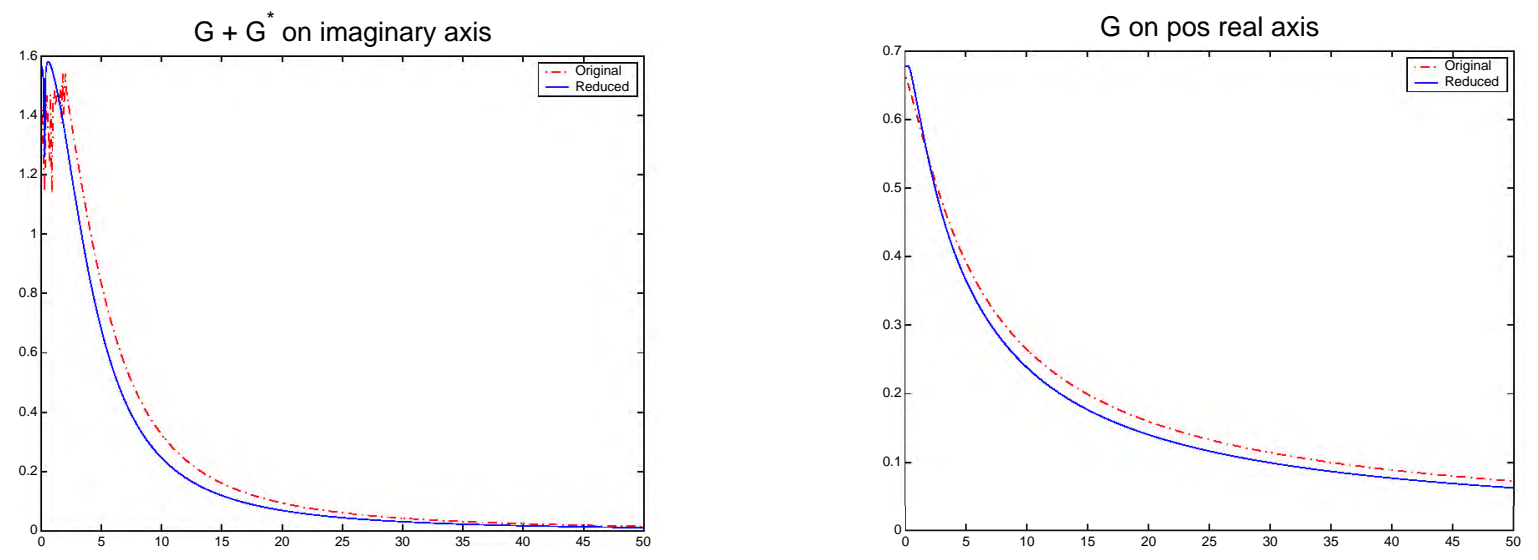

Figure 4: Plot of $\mathbf{G}^{T}(-i \omega)+\mathbf{G}(i \omega) \geq 0$ for $\omega \in \mathbb{R}$ (left) and plot of $\mathbf{G}(s)$ for real positive $s$ (right) .
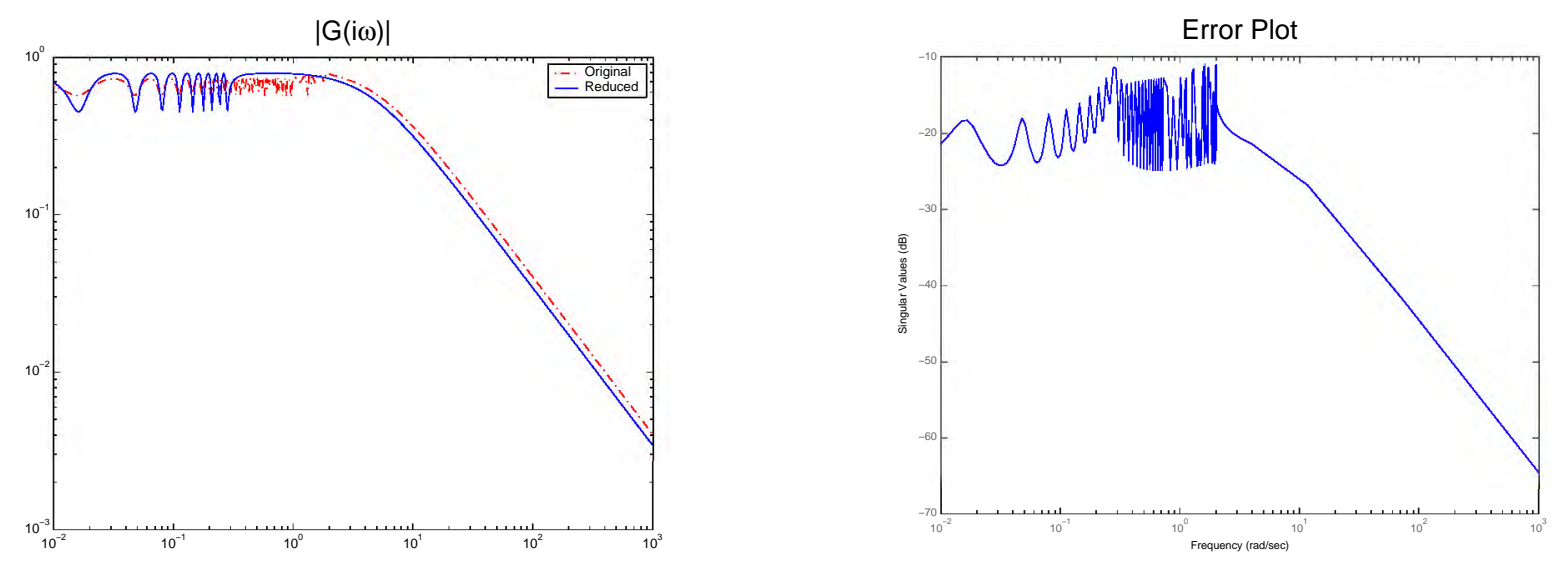

Figure 5: Comparison of absolute value of transfer functions on $i \omega$ axis (left) and sigma plot of the error (right).

approximation properties of balanced reduction. Moreover, the distribution of the spectral zeros for the reduced model are quite different than those selected by the Cayley transformation. The spectral zeros of the reduced model (o) in relation to those of the full system (+) in the right half-plane are shown on the right in Figure 7. The plot for the order 201 system was too cluttered to illustrate the detail, so a smaller RLC system was used for this plot. The distribution of spectral zeros of the smaller system is quite similar to the distribution for the order 201 system. Note that these are non-interpolatory and are in regions that are very different from the previous example.

These results are encouraging, but limited. Additional research and experimentation is needed to better understand this approach. The following questions remain: 1) What is the best choice of interpolation points? 2) Is it possible to derive a bound on the approximation error? 3) What is the best choice for $\mu$ in the Cayley transformation? Future work will include a study of these questions as well as a far more exhaustive set of test examples.

\section{Acknowledgment}

This paper resulted from consideration of the important interpolation result of Thanos Antoulas [2]. The author wishes to thank Professor Antoulas and also Dr. Serkan Gugercin for several enlightening discussions on these results and on the theory of passivity and positive real transfer functions. The author also wishes to thank them for 

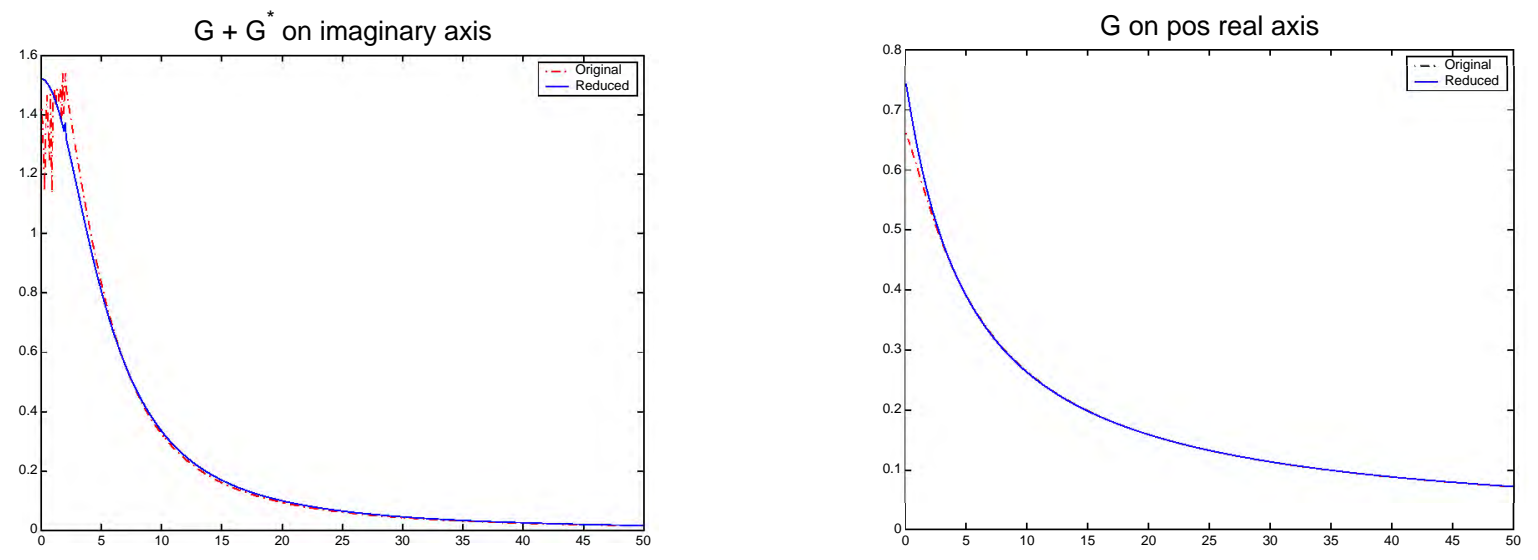

Figure 6: Plot of $\mathbf{G}^{T}(-i \omega)+\mathbf{G}(i \omega) \geq 0$ for $\omega \in \mathbb{R}$ (left) and plot of $\mathbf{G}(s)$ for real positive $s$ (right) .
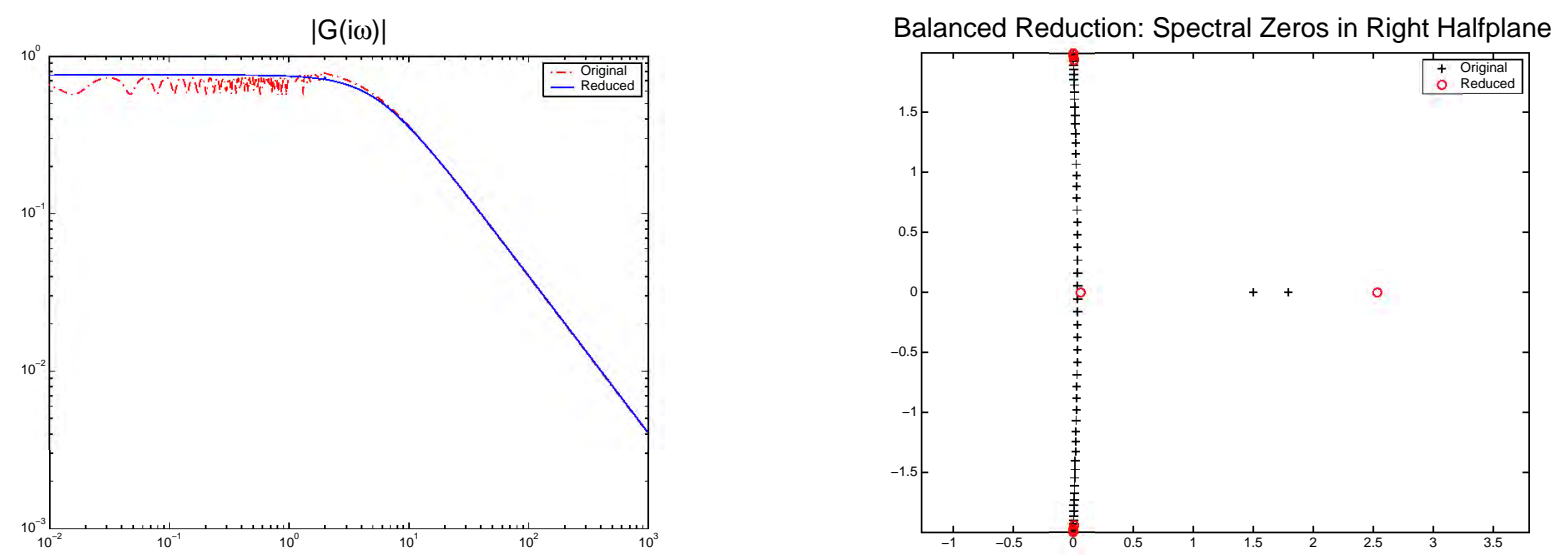

Figure 7: Comparison of absolute value of transfer functions on $i \omega$ axis (left) and Spectral Zero Placement (right).

the RLC circuit example and description.

\section{References}

[1] G.S. Ammar, P. Benner, V. Mehrmann, A multishift algorithm for the numerical solution of algebraic Riccati equations, Electronic Transactions on Numerical Analysis, 1, 33-48, (1993).

[2] A.C. Antoulas, A new result on positive real interpolation and model reduction, Tech. Report, ECE Rice University, November (2002). (First presented at the Householder Meeting in Peebles, July 2002.)

[3] B.D.O. Anderson and S. Vongpanitlerd, Network Analysis and Synthesis: A Modern System Theory Approach, Prentice-Hall, Englewood-Cliffs, New Jersey (1973).

[4] Z. Bai, P. Feldmann and R. Freund, Stable and passive reduced-order models based on partial Padk approximation via the Lanczos process, Bell Laboratories, Lucent Technologies, Numerical Analysis Manuscript 97/3-10, November (1997). 
[5] Z. Bai, P. Feldmann and R. Freund, How to make theoretically passive reduced-order models passive in practice, Proceedings of the IEEE 1998 Custom Integrated Circuits Conference, pages 207-210 (1998).

[6] Z. Bai and R. Freund, Eigenvalue-based characterization and test for positive realness of scalar transfer functions, IEEE Transactions on Automat. Control, AC-45: 2396-2402 (2000).

[7] A. Bunse-Gerstner and V. Mehrmann, The HHDR algorithm and its application to optimal control problems, R.A.I.R.O. Automatique, 23, 305-329, (1989).

[8] R. Byers, A Hamiltonian QR Algorithm, SIAM J. Sci. Stat. Comp. 7, 212-229, (1986).

[9] R. Byers, Solving the algebraic Riccati equation with the matrix sign function, Lin. Alg. Appl. 85, 267-279, (1987).

[10] P. Feldman and R.W. Freund, Efficient linear circuit analysis by Padé approximation via a Lanczos method, IEEE Trans. Computer-Aided Design, 14, 639-649, (1995).

[11] R. Freund, Passive reduced-order models for interconnect simulation and their computation via Krylovsubspace algorithms, Proceedings ACM DAC 99, New Orleans (1999).

[12] S. Gugercin, Projection methods for model reduction of large-scale systems, Ph.D. Thesis (2002).

[13] S. Gugercin and A.C. Antoulas, On balancing related model reduction methods and the corresponding error, Technical Report, ECE Dept., Rice University, September (2002) (submitted).

[14] T. Kailath, Linear Systems, Prentice Hall, Englewood Cliffs, New Jersey, (1980).

[15] A.J. Laub, A Schur method for solving algebraic Riccati equations, IEEE Trans. Automatic Control AC-24, 912-921, (1979),

[16] R. Lehoucq, D.C. Sorensen, and C. Yang, ARPACK Users Guide: Solution of Large Scale Eigenvalue Problems with Implicitly Restarted Arnoldi methods, SIAM Publications, Philadelphia, (1998). (software available at http://www.caam.rice.edu/software/ARPACK)

[17] R. Ober, Balanced parametrization of classes of linear systems, SIAM J. Control and Optimization, 29: 1251-1287 (1991).

[18] A. Odabasioglu, M. Celik, and L.T. Pileggi, PRIMA: Passive reduced-order interconnect macromodeling algorithm, IEEE Trans. Computer Aided Design of Integrated Circuits and Systems, 17: 645-654 (1998).

[19] D.C. Sorensen, Implicit application of polynomial filters in a k-step Arnoldi method, SIAM J. Matrix Anal. Applic., 13: 357-385 (1992).

[20] K. Zhou with J.C. Doyle and K. Glover, Robust and Optimal Control, Prentice Hall, (1995). 